A. Weisbecker, M. Burmester \& A. Schmidt (Hrsg.): Mensch und Computer 2015 Workshopband, Stuttgart: Oldenbourg Wissenschaftsverlag, 2015, S. 577-581.

\title{
Tagungsband des Workshops „Gemeinsam Altern erleben“
}

\author{
Anna Kötteritzsch $^{1}$, Julia Bons ${ }^{2}$, Katja Herrmanny ${ }^{3,4}$, Benjamin Weyers ${ }^{5}$, Ay- \\ segül Dogangün ${ }^{4}$
}

Forschungsgruppe Kooperationssysteme, Universität der Bundeswehr München ${ }^{1}$

Institut Informatik, Hochschule Ruhr West ${ }^{2}$

Forschungsgruppe Interaktive Systeme, Universität Duisburg-Essen ${ }^{3}$

Forschungsgruppe Personal Analytics, Universität Duisburg-Essen ${ }^{4}$

Virtual Reality Group, Rheinisch-Westfälische Technische Hochschule Aachen ${ }^{5}$

\section{Motivation}

Der in Forschungsansätzen zunehmende Gebrauch des Ausdrucks „,alternde Gesellschaft“ weist bereits darauf hin, dass das Altern nicht nur die einzelne Person betrifft, sondern vielmehr ein gemeinsam erlebter Prozess ist, welcher die Person, ihr Umfeld und gesellschaftliche Strukturen beeinflusst. Die Verwendung dieses Ausdrucks birgt aber auch die Gefahr, den Fokus auf den einzelnen Menschen zu verlieren. Eine Herausforderung des Ambient Assisted Living (AAL), das sich mit Unterstützungstechnologien für ältere Menschen befasst, ist die Berücksichtigung beider Aspekte: AAL-Lösungen sollen maßgeschneiderte Systeme sein, die den Anforderungen des Einzelnen gerecht werden, ihm aber gleichzeitig den Kontakt und das gemeinsame Erleben mit anderen Menschen erleichtern und ermöglichen. Die Erkenntnisse des Demografie-Werkstattgesprächs in Berlin „Älter werden bei guter Gesundheit“ (2013) unterstreichen die Relevanz beider Aspekte durch die Bedenken, dass einerseits Kapazitäten für die angemessene gesundheitliche Betreuung fehlen werden, andererseits Altersisolation stark verbreitet sein wird. Die Forschung und Entwicklung im AAL-Umfeld und der Mensch-Technik-Interaktion für den demografischen Wandel haben zum Ziel, mit innovativen und auf die individuellen Bedürfnisse der Nutzer zugeschnittenen Technologien Unterstützung im Alterungsprozess anzubieten und somit die Lebensqualität der Einzelnen in einer sich wandelnden Gesellschaft zu sichern und zu erhöhen.

Gemeinsam Altern erleben heißt in erster Linie, soziale Interaktion und gesellschaftliche Integration auch im Alter zu fördern und somit den negativen Auswirkungen von Einsamkeit im Alter entgegenzuwirken. In der AAL-Forschung und -Entwicklung wird dieses Thema seit 
vielen Jahren fokussiert. Waterworth et al. (2009) entwickelten beispielsweise das System AGNES, das älteren Menschen den Kontakt mit ihren Familien ermöglicht und dafür Ambient Displays und Tangible Userinterfaces bereitstellt. Technologische Entwicklungen werden darüber hinaus auf die Fähigkeiten und Bedürfnisse älterer Nutzer ausgerichtet, um ihnen den Zugang zu digitalen Kommunikationsmedien zu erleichtern und so medial bedingte Isolierung zu verhindern. Dabei müssen die individuellen Ziele der Beteiligten aufgegriffen werden. Gleichzeitig ist es wichtig, mit Hilfe von Technologien die Stärken unterschiedlicher Generationen zu nutzen, sodass alle Generationen von einem Austausch profitieren. Die Notwendigkeit der Schaffung von Technologie, die einen solchen Austausch unterstützt, bildet sich in allen Bereichen des Lebens ab: Im Arbeitsleben, im Wohnen, in Fragen der Mobilität, der Gesundheitsversorgung, sowie im privaten Umfeld. In all diesen Bereichen spielt auch das Erleben von und durch Technik eine entscheidende Rolle. So wird das gemeinsame Erleben von Situationen und Aktivitäten mit Hilfe von technologischen Systemen unterstützt.

Gemeinsam Altern erleben heißt auch gemeinsam Altern gestalten. Forscher aus dem AALBereich stellen heute die Weichen für die Unterstützung des Alterns in den nächsten Jahrzehnten. Der demografische Wandel stellt gesellschaftliche, soziale und wirtschaftliche Anforderungen (auch) an die Gestaltung sozio-technologischer Systeme. Um diesen gerecht zu werden, ist es unerlässlich, Alterungsprozesse und unterstützende Technologien gemeinsam zu gestalten. Nur eine enge Kooperation von Vertretern aus Wissenschaft, Wirtschaft und Endnutzern kann Technologien hervorbringen, die allen Akteuren und den heterogenen Anforderungen gerecht werden. „Gemeinsam“ meint bei der Entwicklung von AAL-Systemen auch interdisziplinäres Arbeiten. Fachrichtungen wie Informatik, Medizin, Pflege oder Psychologie müssen sich vernetzen und kooperativ arbeiten, um Kompetenzen zu bündeln und mehrdimensionale Lösungsansätze zu kreieren. Um also das gemeinsame Erleben des Alterns maßgeblich zu prägen, muss zukünftige AAL-Forschung innovative Konzepte mit unterschiedlichen inhaltlichen und technologischen Ansätzen hervorbringen, welche sowohl den individuellen Menschen als auch die sozio-kulturelle Gesamtstruktur fokussieren. Der Workshop „Gemeinsam Altern erleben“" soll in einem wissenschaftlichen und praxisnahen Diskurs diese zukünftigen Trends identifizieren und Herausforderungen sowie Chancen der gemeinsamen Gestaltung kommender Alterungsprozesse festhalten.

\section{Beiträge}

Bei der Betrachtung der aktuell praktizierten Unterstützung älterer Personen und einer damit etablierten, eher pessimistischen Sichtweise auf das Altern, rücken einige technologische, soziale und wirtschaftliche Herausforderungen in den Vordergrund. Diese umfassen neben Fragen nach den Anforderungen des demographischen Wandels an die Ansprache verschiedener Lebensbereiche durch AAL-Technologien auch die Beeinflussung des Erlebens von Alterungsprozessen und Erfahrungen mit Gruppen von älteren Probanden sowie im intergenerationalen Austausch. Darüber hinaus gewinnen die Fragen nach Risiken und Chancen beim Einsatz von AAL Systemen zunehmend an Bedeutung. 
Marion Gottschalk und Mathias Uslar sprechen in ihrem Beitrag die Herausforderungen der Normung an. Dabei erläutern sie, wie wichtig die Normung für Systeme mit heterogenen Komponenten und Anwendern ist. Gerade bei Systemen im AAL-Bereich, bei denen häufig Komponenten verschiedener Hersteller miteinander kommunizieren, sind Absprachen wie Standards und Normungen unumgänglich. Als gute Mittel zur Unterstützung der Normung sehen sie Anwendungsfälle und Integrationsprofile. In dem von ihnen entwickelten Use Case Management Repository erhalten Entwickler, Forscher und Experten die Möglichkeit gemeinsam Anwendungsfälle zu erarbeiten, zu verifizieren, zu gruppieren und zu kombinieren. Mit Hilfe der so entstandenen Anwendungsfälle und deren Schnittstellenbeschreibungen können dann einfacher Normen ausgewählt werden.

Nils Beckmann et al. betrachten in ihrem Beitrag die Vorteile interdisziplinärer Kooperation in der Verfolgung eines ganzheitlichen Ansatzes im Bereich des Selbstmonitorings. Dabei wird herausgestellt, dass durch den Einbezug von Kompetenzen aus den Bereichen Elektrotechnik, Informatik, Kognitionswissenschaft, Gesundheitswissenschaft und Ethik bestehende Lücken in aktuellen Forschungsansätzen geschlossen werden können. Das von ihnen vorgestellte Projekt PAnalytics soll Informationen zu Alltagsaktivitäten, aus der eigenen Wohn- und Lebensumgebung, manuellen Nutzereingaben und Daten aus sozialen Netzwerken interpretieren, um dem Nutzer Vorschläge für gesundheitsfördernde Aktivitäten anzubieten. Durch die Bereitstellung dessen erzielt das Projekt, ältere Personen in ihrem Erleben des Alterns positiv $\mathrm{zu}$ beeinflussen und einen gesunden Lebensstil zu motivieren.

Anne Grohnert et al. verfolgen ebenfalls einen ganzheitlichen Ansatz in der Rehabilitation und Prävention von Schlaganfällen. Sie erklären in ihrem Beitrag, dass Patienten auch noch nach einem stationären Aufenthalt in einer Klinik Bedarf an therapeutischem Bewegungstraining haben können. Deshalb sei eine flächendeckende Versorgung von Bewegungstherapiemöglichkeiten sehr wichtig, gerade in infrastrukturell schwachen Gebieten und für Patienten, die in ihrer Bewegung eingeschränkt sind. Mit RehaInterAct haben Grohnert et al. ein telemedizinisches Assistenzsystem geschaffen, das es Patienten ermöglicht in ihrem häuslichen Umfeld zu trainieren. Gleichzeitig ermöglicht es medizinischem Personal die Kontrolle über das Training ihrer Patienten auch über weite Entfernungen hinweg auszuüben. Durch den Einsatz von Anwendungen aus dem Bereich Serious Gaming werden zudem Patienten motiviert ihre Übungen regelmäßig durchzuführen.

Sabine Sommer et al. stellen in ihrem Beitrag das Projekt BRELOMATE vor. Dieses Spieleund Kommunikationsportal für ältere Personen erzielt eine Verbesserung der Inklusion älterer Personen durch die Entwicklung von bedürfnisorientierten, leistbaren und einfach bedienbaren Kommunikationstechnologien. Durch die Verfolgung eines nutzerzentrierten Entwicklungsprozesses, welcher die Zielgruppe in die Konzeption und Umsetzung der Plattform einbezieht, konnten die Autoren eine hohe Akzeptanz und Zufriedenheit der Zielgruppe älterer Personen erreichen. Eine weitere Besonderheit liegt in der Nutzung eines zweiten Bildschirms (Second Screen) auf dem Tablet zusätzlich zu der TV-basierten Spiel- und Videokommunikationsübertragung, durch welchen sowohl die Bedienung erleichtert als auch das Erleben des Spielens selbst auf einer weiteren Ebene einbezogen wird. 
Paul März et al. fokussieren ebenfalls den Aufbau einer spielerischen Interaktion, um das gemeinsame Erleben zu gestalten. In einer intergenerationalen Spielsituation werden ältere Personen am eigenen Touchtisch über eine TV-basierte Videokommunikation mit ihren Familienmitgliedern verbunden. In ihrem Beitrag beschreiben die Autoren detailliert die technologische Infrastruktur, die benötigt wird, um eine Echtzeitkommunikation und Interaktion während des Spielens herzustellen. Bei der Betrachtung des Beitrags wird deutlich, dass nur eine reibungslose Funktionsweise der Technologien auch zu der gezielten Unterstützung im gemeinsamen Erleben von Aktivitäten führen kann.

Diese Arbeiten bieten sowohl einen Einblick in den derzeitigen Stand der Forschung und Möglichkeiten der Gestaltung eines Prozesses des gemeinsamen Erlebens als auch eine Grundlage für die Diskussion im Workshop.

\section{Ausblick}

Aufbauend auf den Ergebnissen der AAL-Workshops auf der Mensch und Computer in den Jahren 2011 bis 2014 werden im Workshop „Gemeinsam Altern erleben“ Problemstellungen und Lösungsansätze dargelegt sowie neue Potenziale analysiert, diskutiert und dokumentiert. Ziel ist es, zukunftsfähige Trends des Forschungsbereichs gemeinsam mit Vertretern aus der angewandten Forschung und Wirtschaft sowie mit Endnutzern zu identifizieren und zu erarbeiten. Besonderer Wert wird auf die Interdisziplinarität gelegt, durch die nicht nur Teilnehmende aus dem AAL-Bereich, sondern auch aus angrenzenden Themengebieten zum Austausch beitragen und von den gewonnenen Einblicken in AAL-Technologien, die das Erleben gemeinsamen Alterns unterstützen, profitieren.

Die zuvor beschriebenen Projekte geben bereits einen Ausblick, wie sich AAL-Systeme mit dem Fokus auf das gemeinsame Erleben des Alterns der zentralen Herausforderung zur Sicherung der Rolle(n) älterer Menschen in einer funktionierenden Gesellschaft annehmen können. Die Potenziale von unterstützenden Technologien im Bereich der Integration älterer Menschen in die gesellschaftlichen Strukturen werden bislang von Forschungs- und Entwicklungsvorhaben nicht erschöpft. Durch den Einsatz von innovativen Technologien unter Berücksichtigung der Anforderungen von älteren Menschen, kann ein intergenerationales Erleben des Alterns ermöglicht werden. Beispielsweise ermöglicht der Einsatz der Aufzeichnung von Daten in sozialen Netzwerken die Reflektion gemeinsamer Erlebnisse durch mediale Inhalte (Cornejo et al. 2013), was u.a. in der Biografiearbeit für Menschen mit dementiellen Erkrankungen eingesetzt werden kann. Die Projekte Elders-up (Gil \& Martín 2014) und Expact (Szentivány 2015) inkludieren ältere Personen stärker in soziale Strukturen, indem sie die Expertise von älteren Menschen mit Firmen und besonders Start-ups verknüpfen. Ein weiteres Beispiel für Ansätze, die älteren Menschen eine Teilnahme an sozialer Interaktion ermöglicht, ist das Projekt GetVivid (Moser et al. 2015). Die Autoren erlauben älteren Personen durch den Einsatz von Social-TV-Anwendungen den leichten Zugang zu sozialen Netzwerk Strukturen. Derartige Beispiele zeigen, wie innovative Technologien genutzt werden können, um ein gemeinsames Erleben des Alterns zu gestalten. 
Die Diskussion im Workshop soll Aufschluss geben über die Chancen technologischer Ansätze für ältere Menschen insbesondere in Hinblick auf ein gemeinsames und positives Erleben von Aktivitäten und Erfahrungen bis ins hohe Alter.

Wir freuen uns auf das gemeinsame Erleben eines interessanten und aufschlussreichen Workshops.

\section{Danksagung}

Wir danken den beitragenden Autoren für die Darstellung innovativer Lösungsansätze und die Bereitung der Grundlage für eine angeregte Diskussion. Des Weiteren möchten wir uns beim Programmkomitee für die Unterstützung in der Begutachtung der Beiträge bedanken.

\section{Literaturverzeichnis}

Bundesministerium für Bildung und Forschung (2013). Demografie-Werkstattgespräch in Berlin, Älter werden bei guter Gesundheit. Materialsammlung.

Cornejo, R., Tentori, M., \& Favela, J. (2013). Ambient awareness to strengthen the family social network of older adults. In Computer Supported Cooperative Work (CSCW), 22(2-3), 309-344.

Gil, S. R., Martín, V. S. (2014). ELDERS-UP!. In Computers Helping People with Special Needs. Springer International Publishing. S. 148-151.

Moser, C., Kargl, T., Tscheligi, M., Feldbacher, B., Collini-Nocker, B., Harutunian, M., Schiller, F., Eitelberg, M., Altaani, N., Eisele, M., Osl, P. (2015). GeTVivid: A TV Platform for P2P Support Exchange. In Proceedings of TVX2015, ACM, New York.

Szentivány, C. (2015). Im Fokus - Den Zugang zu Erfahrung und Wissen älterer Generationen eröffnen: Das Projekt „ExpAct - Experience keep people active“. In Andrássy Nachrichten 8. Ausgabe. Andrássy Universität. S. 8-9.

Waterworth, J. A., Ballesteros, S., Peter, C., Bieber, G. et al. (2009). Ageing in a Networked Society Social Inclusion and Mental Stimulation. In Workshop on Affect and Behaviour Related Assistance in Support for the Elderly. Corfu, Greece, June 2009.

\section{Kontaktinformationen}

Gemeinsame E-Mail Adresse der Organisatoren: aal-workshop@kooperationssysteme.de 
